\title{
Caveolin-1 in Breast Cancer: Single Molecule Regulation of Multiple Key Signaling Pathways
}

\author{
Sumadi Lukman Anwar ${ }^{1,2 *}$, Artanto Wahyono', Teguh Aryandono ${ }^{1}$, Samuel J \\ Haryono $^{2,3}$
}

\begin{abstract}
Caveolin-1 is a 22-kD trans-membrane protein enriched in particular plasma membrane invaginations known as caveolae. Cav-1 expression is often dysregulated in human breast cancers, being commonly upregulated in cancer cells and downregulated in stromal cells. As an intracellular scaffolding protein, Cav-1, is involved in several vital biological regulations including endocytosis, transcytosis, vesicular transport, and signaling pathways. Several pathways are modulated by Cav-1 including estrogen receptor, EGFR, Her2/neu, TGFß, and mTOR and represent as major drivers in mammary carcinogenesis. Expression and role of Cav-1 in breast carcinogenesis is highly variable depending on the stage of tumor development as well as context of the cell. However, recent data have shown that downregulation of Cav-1 expression in stromal breast tumors is associated with frequent relapse, resistance to therapy, and poor outcome. Modification of Cav-1 expression for translational cancer therapy is particularly challenging since numerous signaling pathways might be affected. This review focuses on present understanding of Cav-1 in breast carcinogenesis and its potential role as a new biomarker for predicting therapeutic response and prognosis as well as new target for therapeutic manipulation.
\end{abstract}

Keywords: Caveolin-1 - breast cancer - signaling pathway - autophagy - prognosis - therapy

Asian Pac J Cancer Prev, 16 (16), 6803-6812

\section{Introduction}

Caveolae are flask-shaped invaginations of plasma membrane with multiple biological functions. Caveolae play a role as membrane organizing hub and regulate endocytosis, transcytosis, molecular transport, cell adhesion, cell migration, and intracellular signal transduction. (Kiss 2012) In the striated pattern of caveolae, reside several proteins forming a coat of caveolae, which is known as caveolins. With the ability to form homo- and hetero-oligomers, caveolins directly interact with numerous proteins in plasma membrane and are involved in various signaling pathways. (Kiss, 2012) Caveolins consist of 3 family proteins, caveolin-1, -2, and -3 in which caveolin-1 plays a prominent role in cellular transport and communication. Caveolin- 2 is predominantly co-localized with caveolin-1 and forms hetero-oligomers to mediate signal transduction. Caveolin-3 has amino acid sequence highly related to caveolin-1 and is dispersed in different cell types (Gupta et al., 2014).

Caveolin-1 facilitates transport of fatty acid and cholesterol in a lipoprotein chaperone complex. Caveolin-1 also mediates transport of albumin and LDL through transcytosis pathway. Secretion of insulin is mediated by caveolin-1 via ATP dependent-potassium channel and interaction with G-protein coupled receptor located at caveolae. (Mercier and Lisanti, 2012) In addition, caveolin-1 (Cav-1) has been reported to bind to several proteins involved in cell proliferation such as epidermal growth factor receptor (EGFR), Scr protein family members, $\mathrm{H}$-Ras, protein kinase $\mathrm{C}$, and Her2/Neu (Zhang et al., 2013). Caveolin-1 is therefore implicated to play a vital role during initiation and progression of several cancers including breast cancer. (Mercier and Lisanti, 2012; Gupta et al., 2014).

Breast cancer is the most diagnosed cancer among women and emerges as a leading cause of cancer-related mortality and disability. More than 1.38 million new cases are diagnosed every year with annual mortality is around 465,000 (Siegel et al., 2014). Breast cancers develop through multistep progression models involving numerous cellular pathways. Caveolin-1 (Cav-1) has been suggested to play a significant role in breast carcinogenesis (Mercier and Lisanti, 2012; Gupta et al., 2014). Cav-1 expression is deregulated in breast cancer cells and primary breast cancer and stromal tissues (Qian et al., 2011; El-Gendi et al., 2012; Simpkins et al., 2012). Forced expression of Cav-1 in mammary cancer cell lines is able to inhibit oncogenesis and migration. (Sloan et al., 2004). Located at $7 \mathrm{q} 31.1, \mathrm{CAV}-1$ gene is frequently deleted in breast

${ }^{1}$ Department of Surgery, Faculty of Medicine, Universitas Gadjah Mada, Yogyakarta, ${ }^{2}$ Mochtar Riady Comprehensive Cancer Center (MRCCC) Siloam Hospital Semanggi, Jakarta, ${ }^{3}$ Department of Surgical Oncology, National Cancer Center of Dharmais Hospital, Jakarta,Indonesia*For correspondence: sl.anwar@ugm.ac.id 
cancer. (Williams and Lisanti, 2004) For the first time in 2001, Hayashi et al. reported that Cav-1 proline-to-leusine substitution (P132L) was documented in $16 \%$ primary breast cancer specimens (Hayashi et al., 2001). However, Cav-1 has also been implicated as oncogenic driver in breast cancer. Transfection of Cav-1 in breast cancer cell lines induced growth and colony formation ( $\mathrm{Wu}$ et al., 2007). Amplification and overexpression of Cav-1 have also been reported in primary breast cancer (Savage et al., 2007). The dual roles of Cav-1 during breast development are suggested to occur specifically according to different progression steps of oncogenesis (Gupta et al., 2014).

Recent studies have shown that Cav-1 is particularly useful marker for diagnosis, prognosis and predictive therapeutic outcome. In a large meta-analysis, Ma et al. demonstrated that diminished Cav-1 expression in stromal cells is significantly associated with poor breast cancer outcome (Ma et al., 2013). In invasive micropapillary carcinoma, a form of breast cancer with abundant stromal cancer associated fibroblasts (CAFs) and high propensity for nodal metastasis and worse outcome, expression of stromal Cav-1 is significantly lower than in invasive ductal carcinoma (Ren et al., 2014).

The role of Cav-1 in breast carcinogenesis is facilitated through interaction with Src family proteins, H-Ras, epidermal growth factor receptor (EGFR), HER2, estrogen receptor, p85 regulatory subunit of MAPK cascade, and endothelial nitric oxide synthase (eNOS). (Park et al., 2009; Mercier and Lisanti, 2012; Gupta et al., 2014) Cascade of downstream kinases requires interaction of those proteins in which with caveolin-scaffolding domain (CFD) functions as attachment site for restrained conformation. These data show that Cav-1 protein plays an important role in the initiation and progression of breast cancer through different cellular pathways. However, the role of Cav-1 in breast carcinogenesis is still controversial either as oncogenic or suppressor protein. In addition, breast cancer is a very heterogeneous disease consisting of several subclasses and involving a wide variety of molecular and cellular pathways with different treatment options, prognosis, and clinical outcome (Witkiewicz et al., 2009; Mercier and Lisanti, 2012). This review will comprehensively discuss the biological functions and their role of Cav-1 in the pathogenesis of breast cancer and its potential for translational applications in the breast cancer management.

\section{Molecular Structure and Biological Functions of Cav-1}

Caveolin-1 (Cav-1) has been initially reported as a phosphoprotein substrate of Src kinase. Further studies showed that Cav-1 protein is predominantly enriched in caveolae, a unique type of lipid raft that is responsible for signal transduction. (Williams and Lisanti, 2004; Lajoie P, Nabi IP, 2010) Molecular structure of Cav-1 resembles as a hairpin with $\mathrm{N}$ - and $\mathrm{C}$-terminal ends. In an active form, Cav-1 is usually phosphorylated on tyrosine-14 (Y14) and / or serine-80 (Shajahan et al., 2012). Kinases such as Src, Fyn, Yes, and c-Abl are able to induce phosphorylation on Y14 leading to oligomerization and activation of scaffolding domain to further stimulate interaction with other proteins including $G$ proteins, phospholipases, protein kinase $\mathrm{A}$, protein kinase $\mathrm{C}$, nitric oxide sunthetases, adenylate cyclases, tyrosine kinase receptors and Ras family GTPases (Martinez-Outschoorn et al., 2015). Phosphorylation occurs constitutively or promptly in response to growth factor receptor or integrin activation. In addition, phosphorylation of serine 80 is suggested to regulate cholesterol transport (Fielding et al., 2004). The C-terminal acts as a domain to localize Cav-1 at the plasma membrane while the scaffolding domain facilitates direct interaction with cholesterol regulating raft organization and cholesterol trafficking (Tagawa et al., 2005).

One of the most remarkable findings is the ability of Cav-1 to regulate some tyrosine kinase receptors, either as inhibitor or activator depending on the structure of tyrosine kinase receptors and signaling patterns. Cav-1 negatively regulates EGFR receptors while positively regulates insulin receptors (Park et al., 2000; Cohen et al., 2003). Different from EGFRs that consist of single transmembrane monomer and form dimer upon stimulation; insulin receptors consist of extracellular $\alpha$-subunit and trans-membrane $\beta$-subunit that form dimers and are connected by disulphide bonds. After phosphorylated, EGFRs and insulin receptors recruit different proteins to transmit subsequent signals (Park et al., 2000; Senetta et al., 2013).

Inactivated EGFRs are clustered within caveolae and leave this lipid raft structure upon activation. Evidence using electron microscopy showed that EGFR disappears from caveolae if EGF stimulation is absent (Mineo et al., 1999; Senetta et al., 2013). Raft internalization is regulated by Cav-1 scaffolds that indirectly regulate EGFR (Williams and Lisanti, 2004; Lajoie P, Nabi IR, 2010). In addition, oligomeric Cav-1 domains bind to inactive EGFR and prevent its activation (Park et al., 2000). Internalization of EGFR usually occurs at high Cav-1 concentration with low EGF stimuli and is mediated through clatrin-dependent pathway (Maldonado-Báez et al., 2013). Ratio EGF/EGFR levels also determine plasma membrane compartmentalization of active EGFR (Burke et al., 2001; Lajoie and Nabi, 2010).

On the other hand, Cav-1 has also been reported to promote EGFR signaling leading to cell proliferation and migration (Agelaki et al., 2009). How Cav-1 regulates EGFR signaling resulting in activation or inhibition depends on the affected pathways. Cav-1 inhibits EGFR pathway mediated by Grb2-Sos-Ras and ERK1/2 but stimulates PI3K pathway (Park et al., 2000; Mercier and Lisanti, 2012). In addition, Cav-1 directly interacts with ERK1/2 via scaffolding domain to prevent activation. Interaction of Cav-1 with Akt as downstream kinase of PI3K pathway induces EGFR activation leading to elevated cell survival (Park et al., 2009). Activation EGFR by EGF will stimulate Src activation to further phosphorylate Cav-1.EGF is also able to activate caveolae formation through phosphorylation of tyrosine-14 leading to greater responses such as endocytosis and caveolae fusion (Park et al., 2009; Mercier and Lisanti, 2012; Sotgia et al., 2011). Thyrosine 14 phosphorylation also 
causes downfall of cell-cell contacts. Long term EGF exposure affects Cav-1 and E-cadherin downregulation that will promote migration through various kinases including ERK1/2, FAK, and PI3K (Park and, Han, 2009). In addition, phosphorylated Cav-1 also induces NGF and TGF $\beta$ signaling pathways. (Park and, Han, 2009).

\section{Controversial Role of Caveolin-1 During Breast Carcinogenesis}

The first evidence showing Cav-1 as a tumor suppressor was described by Lee et al. (1998) Re-expression of Cav-1 in human breast cancer cells results in attenuation of cell growth (Lee et al., 1998). Functional assays using MCF7 breast cancer cells showed diminished proliferation and cell viability after transfection with CAV-1 gene. (Hino et al., 2003) Cav-1 is able to suppress malignant phenotypes by inhibiting cell division, cell migration, and invasion (Fiucci et al., 2002). Haploinsufficiency of Cav-1 in MCF10A allows cell growth although is not adequate for transformation and/or reversion (Zou et al., 2003; Mercier and Lisanti, 2012). Loss of Cav-1 function is mainly related to the abrogation of Thyrosine-14 or Serin-80 phosphorylation (Mercier and Lisanti, 2012; Senetta et al., 2013).

Re-expression of Cav-1 in breast cancer cell line leads to up-regulation of BRCA1 protein, on the other hand, BRCA1 stimulates Cav-1 expression through promoter induction (Glait et al., 2006; Wang et al., 2008). In human breast cancer, expression of Cav-1 is variably reported. Lower Cav-1 expression has been correlated with progression of invasive lobular carcinomas (ILCs) (Perrone et al., 2009). Further study using microarray and immunohistochemistry revealed low Cav-1 expression in ILCs irrespective to histological grade and ER status and also in invasive ductal carcinomas (IDCs) (Weigelt et al., 2010). Breast cancers with ER expression both ductal and lobular express particularly low levels of Cav-1 (Mercier and Lisanti, 2012).

The role of Cav-1 as tumor suppressor is highlighted by studies showing inactivated mutation resulting in proline-to-leucine substitution (Cav-1P132L) that affects $\sim 14 \%$ of total cases exclusively in ER $\alpha$-positive breast cancer (Hayashi et al., 2001). It is indicated that Cav1P132L mutation is found more frequently in breast cancer cases with relapse. (Mercier and Lisanti, 2012) Cav-1P132L is suggested to function as dominant negative mutation that can impair oligomerization with misfolding and mislocalization within golgi complex (Lee et al., 2002). However, Cav-1P132L is not sufficient to transform cells into malignant cells. Other mechanisms as second hits might be required. Recent report showed hypermethylation of Cav-1 promoter and correlated negatively with its expression (Rao et al., 2012). However, analysis in 82 fresh breast cancer tissues, 158 paraffin blocks, and 39 breast cancer cell lines revealed low frequency of Cav-1 mutations including P132L (Patani et al., 2012). In addition, a spectrum of CAV-1 mutations was reported in specific Kashmir population and was implicated to play a role in the breast cancer progression (Syeed et al., 2010).
On the other hand, Cav-1 can serve as a pro-survival protein by regulating several pathways including MAP Kinase p38 and the downstream Akt, PI3K, ERK1/2, as well as Bcl-2 (Fujita et al., 2004; Williams et al., 2004; Mercier and Lisanti, 2012). It is indicated that biological pathways regulated by Cav-1 are tissue and cell specific. In cancer cells, Cav-1 promotes cell survival and inhibits cell death through upregulation of IGF1 signaling(Williams TM, Lisanti MP, 2005), interaction with PP1 and PP2A phosphatases to activate Akt and ERK1/2 and induction of TRAIL receptor. (Williams and Lisanti, 2005; Mercier and Lisanti, 2012; Park and, Han, 2009)

Breast cancer cells often express high Cav-1 compared to normal breast epithelium (Eynden et al., 2006; Qian et al. 2011). However, Cav-1 overexpression solely is not sufficient to induce transformation into breast cancer cells. (Mercier and Lisanti, 2012) Cav-1 has also been implicated in mediating multi-drug resistance (MDR) including into Adriamycin (Lavie et al., 2001). Several studies showed that Cav-1 expression correlated with shorter diseasefree survival and overall survival (Savage et al., 2007; Mercier and Lisanti, 2012). High expression of Cav-1 has been observed in metastatic breast cancer with significant poorer outcome (Savage, et al. 2007; Sotgia, et al., 2011; Mercier and Lisanti, 2012). Expression of Cav-1 is positively correlated with histological grade, expression of EGFR, cytokeratins 5/6, 14 and 17, as well as p53. Inverse correlation is observed between expression of Cav-1 and ER, PR, Her2, and cyclin D1. In addition, Cav-1 positive breast cancers are mostly basal-like phenotype. Cav-1 expression is higher in familial breast cancers compared to the sporadic cases (Savage, et al. 2007; Witkiewicz et al., 2009). Almost all metaplastic breast cancers express Cav-1. Gene amplification has also been observed in breast cancers with Cav-1 overexpression (Fiucci et al., 2002; Savage et al., 2007).

It is suggested that during carcinogenesis, Cav-1 plays either as tumor suppressor or oncogenic depending on the stage of tumor development (Gupta et al., 2014). In the beginning of tumor formation, Cav-1 plays as a tumor suppressor. With the complex tumor metabolism and stromal role in the tumor development, Cav-1 expression is elevated in cancer cells while decreased in stromal cells. However, the molecular processes underlying functional switch of Cav-1 during breast oncogenesis still need to be revealed.

\section{Caveolin-1 in Autophagy and Cancer Metabolism}

Epithelial cancer cells are able to induce aerobic glycolysis through activation of fibroblasts surrounding the cancer cells ('Reverse Warburg Effect'). The significant roles of tumor stroma in the tumor development have been well described; however, the detailed cellular process still has to be delineated. (Cirri and Chiarugi, 2011) Due to aerobic glycolysis induced by cancer epithelial cells, stromal fibroblasts differentiate into myofibroblasts that can produce lactate as well as pyruvate as a result of mitochondrial autophagy within myofibroblast. Subsequently, cancer cells will take these energy-rich 
metabolites (lactate and pyruvate) into their mitochondrial system to further provide such an efficient energy resource to promote cell proliferation and tumor growth (Cirri and Chiarugi, 2011; Sotgia et al., 2012). Cancer stroma will therefore directly facilitate tumor development, migration and angiogenesis.

Fibroblasts and myofibroblasts forming tumor stroma are known as cancer-associated fibroblasts (CAFs). Myofibroblasts express surface markers commonly shown by muscle cells and pericytes and form the main cell population of stromal CAFs (Otranto et al., 2012). In addition, in vitro and in vivo studies have demonstrated that cancer cell proliferation can be significantly enhanced by addition of cancer-associated fibroblasts (CAFs) (Otranto et al., 2012). On the other hand, normal fibroblasts is generally not be able to promote tumor growth (Bhowmick et al., 2004). Inhibition of CAFs is related to diminished tumor progression (Bhowmick et al., 2004). CAFs have an ability to proliferate faster than normal fibroblast and share similar characteristics with fibroblasts located in keloids (Bhowmick et al., 2004; Dong et al., 2013).

Cav-1 has been reported as a significant regulator of stromal remodeling to support cancer growth and metastasis. (Mercier and Lisanti, 2012) Cav-1 regulates p190RhoGAP resulting in cell ellongation, matrix alligning, and microenvironment stiffening (Goetz et al., 2011). CAFs derived from invasive breast tumors show reduced Cav-1 expressions, increased pRB expression and elevated BrdU incorporation, as well as increased cell proliferation. Re-expression of Cav-1 in CAFs results in inhibition of cell proliferation through modulation of RB pathway (Mercier et al., 2008). In addition, CAFs with low Cav-1 expression showed a unique gene expression profile similar to RB1 deficient cells (Mercier et al., 2008). Moreover, loss of stromal Cav-1 expression in breast cancer tissues is associated with frequent recurrence, resistance to hormonal therapy, and lymph node infiltration (Witkiewicz et al., 2009). The prognostic value of reduced stromal Cav-1 expression is not related with current prognostic markers including ER, PR, and Her2. In addition, diminished stromal Cav-1 expression in early breast cancer lesions such as DCIS can be used as predictor for progression into invasive breast cancer (Witkiewicz et al., 2009). Involving more than 350 breast cancer patients, Simpkins et al. (2012) reported that decreased stromal Cav-1 expression was correlated with lower disease-free survival. Similarly, El-Gendi et al. inferred that absence of stromal Cav-1 correlated with advance tumor stage, higher recurrence rate, and shorter progression free breast cancer survival (El-Gendi et al., 2012). In particular, elevated tumor Cav-1 expression and decreased Cav-1 stromal could determine sub-population of breast cancer patients with potentially have worse clinical outcome (Qian et al., 2011).

How stromal Cav-1 expression affecting poor breast cancer prognosis is still need to be determined. Using wild-type and Cav-1 deficient stromal fibroblasts, Bonuccelli et al. showed that fibroblasts with lack of Cav-1 expression were able to further promote tumor growth and angiogenesis through activation of glycolytic enzymes (Bonuccelli et al., 2010). Cav-1 depletion causes mitochondrial impairment, oxidative storms, and aerobic glycolysis in cancer associated fibroblasts. Damaged mitochondria due to oxidative stress are then cleared from cancer-associated fibroblasts through autophagy (Pavlides et al., 2012; Jezierska-Drutel et al., 2013). Therefore, stromal fibroblasts can deliver nutrients including lactate to stimulate mitochondrial synthesis and aerobic metabolism in cancer cells (Pavlides et al., 2012; Jezierska-Drutel et al., 2013). Moreover, oxidative stress in stromal fibroblasts affects genomic stability of the adjacent cancer cells (Jezierska-Drutel et al., 2013). Reactive oxygen producing fibroblasts are able to dowregulate Cav-1 (Jezierska-Drutel et al., 2013). In addition, stromal fibroblasts with Cav-1 depletion were able to induce upregulation of TIGAR in the epithelial cancer cells conferring protection of cancer cells from cell death (Martinez-Outschoorn et al,. 2010). In Cav-1 (-/-) null mice, some metabolites including ADMA (asymmetric dimethyl arginine) and BHB (keton body) are upregulated in mammary fat pads due to oxidative stress, mitochondrial damage, and autophagic dysregulation. Some cancer-associated microRNAs (miR-31 and miR-34c) are upregulated in which these microRNAs are associated with HIF1 $\alpha$ and authophagic signaling pathways. Therefore, induction of hypoxia and autophagy by tumor cells to stromal compartments results in Cav-1 downregulation and subsequently induces metabolic imbalance that support tumor growth and metastasis (Pavlides et al., 2010). Co-injection of Cav-1 depleted fibroblasts with breast cancer cells MDA-MB-231 increased tumor volume by almost 4-fold. Low Cav-1 expression in stromal fibroblasts resulted in the upregulation of genes involved in myofibroblast differentiation and oxidative stress or hypoxia. The carcinogenic potential of Cav-1 depleted fibroblasts could be inhibited by superoxide dismutase 2 (SOD2) (Trimmer et al., 2011). Triple negative, basal-like, and tamoxifen-resistance in ER $\alpha$ positive breast cancers are associated with Cav-1 deficient stromal fibroblasts in the tumor micro-environment that can drive into poorer clinical outcome (Trimmer et al., 2011). Caveolin emerged as a marker for highly predictive of metastatic risk in breast cancer (Giusiano et al., 2011). In addition, using laser microdissected breast cancer-associated stroma, Witkiewicz et al. were able to characterize signaling pathways that were activated in Cav-1 depleted stroma. Subsequent analysis showed that enrichment of these genes are functionally involved in stemness, inflammation, DNA damage, aging, oxidative stress, hypoxia, and autophagy (Witkiewicz et al., 2011).

Cav-1 depleted stroma can induce inflammation through oxidative stress and activation of NFxB. Autophagic microenvironment also induces secretion of several inflammatory mediators (including IL-6, IL-8, IL-10, MIP1a, IFN $\gamma$, RANTES (CCL5), and GMCSF. These inflammatory mediators are able to induce onset of autophagy in fibroblasts and attract inflammatory cells into the stroma (Martinez-Outschoorn et al., 2011). In Cav-1 negative fibroblasts, plasminogen activator inhibitor type 1 and type 2 (PAI-1 and PAI-2) are significantly upregulated resulting in increased autophagy and mitochondrial activity (Castello-Cros et al., 2011). Absent Cav-1 
expression in stromal tissues of invasive ductal carcinoma (IDC) significantly correlated with high tumor stage, high nodal stage, high cancer stage, ER negativity, and tumor recurrence. Loss of Cav-1 expression was also associated with a shorter disease-free survival and overall survival IDC (Koo et al., 2011).

To reveal signaling pathway by which depletion of Cav-1 drives tumor-promoting microenvironment and poor breast cancer outcomes, Castello-Cros performed proteomic and transcriptional profiling and demonstrated that plasminogen activator inhibitor type 1 and type 2 (PAI-1 and PAI-2) expression is significantly increased in Cav-1(-/-) fibroblasts. Moreover, fibroblasts with PAI-1 and -2 expression promote cell proliferation, metastasis, autophagy and mitochondrial biogenesis in the surrounding cancer cells (Castello-Cros et al., 2011). The supporting system provided by stromal tissues in carcinogenesis arises from metabolic coupling in which oxidative stress caused by aerobic glycolysis and mitochondrial dysfunction within stroma is required for cancer initiation and progression. Stromal oxidative stress induces DNA damages and mutagenesis of cancer cells. In addition, autophagy in the tumor stroma is able to further interrupt energy balance of cancer cells. Glutamine addiction of cancer cells results in the production of ammonia which is important for maintenance of autophagy in the stroma. Loss of stromal Cav-1 expression is an indicator for the occurrence of hypoxia, oxidative stress, and autophagy within tumor microenvironment (Sotgia et al., 2011). In the metabolic regulation, microRNA has also been associated with regulation of Cav-1 expression. Upon caloric restriction, miR-203 is highly upregulated while Cav-1 is downregulated. This implicates the role of microRNA in the regulation of Cav-1 expression and metabolic imbalance during cancer formation (Ørom et al., 2012).

Despite large amount of data showing that stromal Cav-1 downregulation in breast cancer were associated with poor prognosis, Goetz et al. indicated that stromal Cav-1 expression was correlated with low breast cancer survival (Goetz et al., 2011). Different methodologies, cutoff-point for Cav-1 expression, and tumor heterogeneity might cause these discrepancies.

\section{Caveolin-1 in ER Pathway During Breast Carcinogenesis}

Estrogen signaling pathway has been established as a factor associated with breast carcinogenesis. Prolonged exposure to estrogen such as in women with early menarche, late menopause, and hormonal replacement therapy correlates significantly with elevated risk of breast cancer. (Clemons M, Goss P, 2001) Binding of estrogen to estrogen receptor alpha $(\mathrm{ER} \alpha)$ induces receptor conformation change and downstream ER-pathway activation leading to increased cell proliferation (Carroll et al., 2006). Approximately, 10-15\% of normal breast epithelial cells express ER $\alpha$. This receptor is commonly upregulated in breast cancer making nearly $75 \%$ invasive breast cancer cases are ER $\alpha$-positive. Several genes and transcripton factors are activated upon estrogen-ER $\alpha$ binding including AP1, SP1, and NFxB leading to elevated cell proliferation (Carroll et al., 2006). Anti-estrogen has emerged as the first targeted therapy that revolutionizes cancer management in general. Hormonal therapy using tamoxifen (an anti-estrogen) for 5 years in breast cancer has been successfully improved overall survival. However, almost $50 \%$ of breast cancer patients sooner or later develop resistance and the tumor can recur after anti-hormonal therapy. Recent studies have demonstrated that Cav-1 acts as regulator of ER $\alpha$ expression (Schlegel et al., 1999; Sotgia et al., 2006).

Cav-1 has emerged as an important regulator of ER $\alpha$ expression as well as response to anti-estrogen (Schlegel et al., 1999; Sotgia et al., 2006). In mammary epithelial cells, Cav-1 depletion leads to ER $\alpha$ up-regulation and correlates with anchorage-independent growth (Zou et al., 2003). Therefore, Cav-1 functions indirectly as negative regulator of cell proliferation mediated by ER $\alpha$-pathway. Cav-1 knock-out mice develop mammary hyperplasia. Bilateral ovariectomy in Cav-1 knock-out mice abolishes the hyperplasia (Witkiewicz et al., 2009). Accelerated mammary gland development has also been shown in Cav-1 deficient mice in addition to premature lactation and hyperactivation of Jak-2/STAT5a signaling (Park et al., 2002).

In addition, expression of Cav-1 in breast cancer cells causes ligand-independent concentration of ER $\alpha$ and induces ER-response element (Schlegel et al., 2001). Co-precipitation of Cav-1 and ER $\alpha$ has also been shown by Schlegel et al. (1999) Cav-1 interacts through its scaffolding protein (residues 82-101) to residues 1-282 of ER $\alpha$ which contain AF-1 (Activation Function-1) and DNA binding domain. AF-1 is activated mainly by phosphorylation and co-expression with Cav-1 potentiated ER $\alpha$ phosphorylation (Ser-118) through ERK1/2independent pathway (Schlegel et al., 2001). Study in primary breast cancer tissues showed that expression of Cav-1 protein and mRNAs are generally reduced in tumor cells. Downregulation of Cav-1 is associated with increasing tumor size and ER and PR negativity (Sagara et al., 2004). In addition, haplosuffiency of Cav-1 resulted in increased expression and activation of ER $\alpha$. Transient depletion of Cav-1 using beta-methyl-cyclodextrin also induced ER $\alpha$ upregulation. Addition of estradiol (E2) has also been able to accelerate anchorage-independent growth in vitro and in the nude mice (Schlegel et al., 1999; Sotgia et al., 2006; Mercier and Lisanti, 2012).

\section{Caveolin-1 and Her2/ERBB2 Signaling Pathway}

Both CAV1 and CAV2 genes reside at the fragile region of 7q31 (FRA7G), and deletion or loss of heterozygosity at this region has been associated with breast cancer (Sagara et al., 2004). Using primary breast cancer specimens, Park et al. showed that reduced Cav-1 expression correlated inversely with HER2 and EGFR expression in invasive ductal mammary carcinoma (Park et al., 2005). In addition, Cav-1 expression is linked to the basal-like breast cancer, a subtype that usually does not express Her2 (Rodríguez Pinilla et al., 2006). Sagara et al. reported significant 
Sumadi Lukman Anwar et al

association between low Cav-2 expression with negative hormonal receptor (ER, PR) and high Her-2 expression in breast cancer but no correlation was found between Her-2 and Cav-1 mRNA levels (Sagara et al., 2004). In addition, Cav-1 expression was associated with increasing tumor size (Sagara et al., 2004). However, elevated expression of Cav-1 in the adjacent stromal tissues is correlated with less metastasis and better overall survival (Sloan et al., 2009). In Her2 positive breast cancer, presence of Cav-1 in the microenvironment tissues is suggested to modulate tumor development (Sloan et al., 2009). Recent study using breast cancer cells and proteomic analysis by Zhang et al. indicated that Cav-1 was involved in the EGFR and greater ERBB2 signaling (Zhang et al., 2013). Wang et al. showed that in mammary epithelial cells, Cav-1 can negatively regulate TLR4 and play a crucial role in the activation of MAPK pathway (Wang et al., 2013). Since Cav-1 is inversely correlated with Her-2 expression; it indicates the possible role of oncogenic activity in Her-2 negative breast cancer through EGFR and MAPK pathway. In the presence of both Cav-1 and Her-2 expression, breast cancer cells might develop resistance to trastuzumab (monoclonal antibody against Her-2 that is commonly used for treatment of Her-2 positive breast cancer). Cav1 expression is suggested to induce internalization and endocytosis of Her2-trastuzumab complex (Sekhar et al., 2013).

\section{Cav-1 Expression and Predictive Therapeutic Responses in Breast Cancer}

As Cav-1 regulates some vital biological pathways in breast cancer, the differential expression is associated with therapeutic outcomes. Elevated Cav-1 expression is suggested to induce resistance against trastuzumab (Sekhar et al., 2013). In subpopulation of breast cancer stem cells, expression of Cav-1 is upregulated particularly after treatment with chemotherapy. In breast cancer stem cells, Cav-1 expression diminishes $\beta$-catenin/ABCG2 signaling and is responsible for chemoresistance (Wang et al., 2014). Using breast cancer cells, Thomas et al. showed that loss of Cav-1 expression was associated with resistance to tamoxifen. Therefore, expression of Cav-1 is probably indicator for response to hormonal therapy using tamoxifen (Thomas et al., 2010) Cav-1 interacts with $\mathrm{ER} \alpha$ to subsequently reduce PKC $\alpha$ expression. Tamoxifen resistance in breast cancer is often mediated by overexpression of PKC $\alpha$ (Tian et al., 2009; Perez et al., 2013). Inhibitor of mammalian target of rapamycin (mTOR) has emerged as a new targeted therapy in breast cancer (Zagouri et al., 2012). Using mouse models, Mercier et al. showed that reduction of Cav-1 expression in stromal (microenvironment) breast tumor was related to response to mammalian target of rapamycin (mTOR) inhibitor (Mercier et al., 2012). In addition, Shajahan et al. showed that phosphorylation of tyrosine in caveolin-1 (Tyr-14) increases sensitivity to paclitaxel (Shajahan et al., 2012). Paclitaxel is an effective anti-microtubule agent for breast cancer. In ER-positive breast cancer, Tyr-14 phosphorylation facilitates mitochondrial apoptosis by inhibiting BCL2 and BCLxL proteins via
c-Jun N-terminal kinase (Shajahan et al., 2012). Using 39 different breast cancer cell lines, Finn et al., evaluated efficacy of desatinib, a small molecule inhibitor of Src and Abl kinases. Basal subtype were particularly the most sensitive and upregulation of Cav-1 (together with moesin and yes-associated protein1) was revealed as predictive marker for clinical response upon desatinib treatment (Finn et al., 2007).

\section{Perspective for Translational and Clinical Application of Cav-1 in Breast Cancer}

The importance of Cav-1 dysregulation in breast cancer is limited in research settings and is not yet corroborated for application as diagnostic marker and predictive markers in the clinics. Cav-1 has been revealed to play an important role in breast cancer development. Cav-1 mediates regulation of metabolic balance between glycolytic stromal tumor and oxidative cancer cells. Downregulation of Cav-1 and upregulation of MCT4 appear as novel biomarkers for Warburg effect, metabolic synergy, as well as prognostic marker in breast cancer (Martinez-Outschoorn et al., 2014). In addition, downregulation of Cav-1 expression in stromal cells predicts early cancer recurrence, lymph node infiltration, and chemotherapeutic resistance almost in all subtypes of breast cancer. Loss of caveolin is also strongly associated with poor outcome and tamoxifen resistance. Especially in triple negative breast cancer, overall survival is significantly higher in patients with stromal Cav-1 expression in comparison to those with low Cav-1 expression (Elsheikh et al., 2008; Witkiewicz et al., 2009). Expression of Cav-1 has been associated with resistance to trastuzumab, a monoclonal antibody against Her2/neu as well as small molecule tyrosine kinase inhibitor, desatinib (Finn et al., 2007; Sekhar et al., 2013). In inflammatory breast cancer (IBC), a rare subtype of breast cancer with very aggressive behavior and poorest clinical outcome, Cav-1 is significantly upregulated (Van Den Eynden et al., 2006). To differentiate breast cancer with inflammatory reaction and specific type of IBC, expression of Cav-1 might be useful marker. It is believed that Cav-1 overexpression in IBC affects RhoC switch and Akt1 phosphorylation to mediate invasion and metastasis (Joglekar et al., 2015).

Translating dysregulation of Cav-1 expression in primary breast cancer tumors and stroma as clinical markers needs standardization of technical protocols. Most studies of expression Cav-1 analysis use immunohistochemistry to evaluate Cav-1 expression (Mercier and Lisanti, 2012; Patani et al., 2012). Universal protocols, specific antibodies and scoring systems have to be formulated to ensure reliability and avoid variability among different health centers. More robust techniques such as qRT-PCR and microarray after laser capture micro-dissection might increase specificity to differentiate cancer and stromal cells. However, these techniques are time consuming and not always available in clinical laboratories.

Modulation of Cav-1 expression tends to affect multitude biological pathways (Schlegel et al., 1999; Park et al., 2005; Bonuccelli et al., 2010). Therefore, therapeutic 


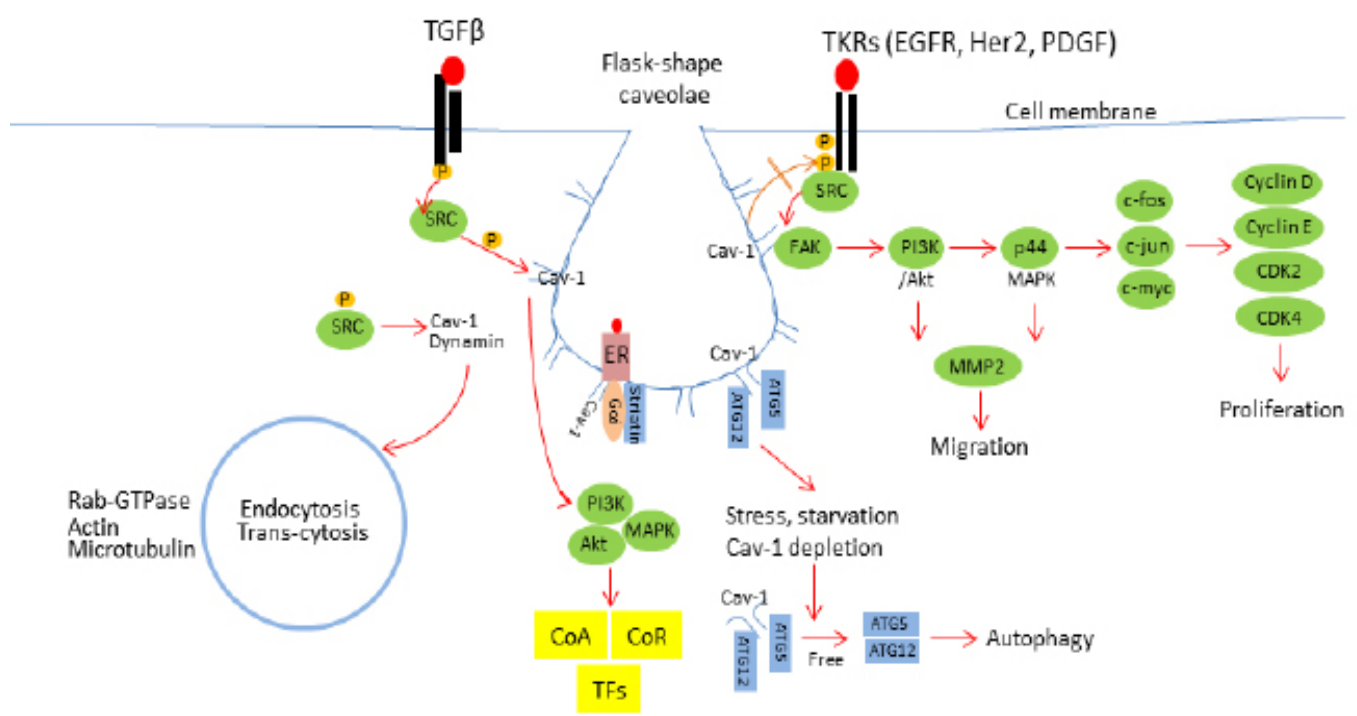

Figure 1. Cav-1 Regulates Several Signaling Pathways in Breast Carcinogenesis Including ER (estrogen receptor), Tyrosine Kinases (EGFR, Epidermal-like Growth Factor Receptor; Her2/neu, Insulin-like Growth Factor; and PDGF, Platelet Derived Growth Factor), TGF- $\beta$ (Transforming Growth Factor- $\beta$ ), autophagy, and endocytosis, transcytosis, as well as molecular transport. Downstream proteins such as Src, PI3K, MAPK as well as transcription factors are activated upon dysregulation of Cav-1 expression and activation

window that will be achieved from modulation of Cav-1 expression needs to be carefully designed. Inhibition or forced expression in specific cell population is useful for cancer therapy. Cav-1 mimicking peptides have tumor suppressor effects as well as anti-angiogenesis (Williams et al. 2004). These peptides have been reported to modulate inflammatory response by inhibiting nitric oxide (Bucci et al., 2000). Overexpression using Cav-1 mimics inhibits Her2 autophosphorylation and other kinase function. (Cai C, Chen J, 2004) In tumor microenvironment especially fibroblast associated tumor, Cav-1 is downregulated. Chloroquin and other autophagy and lysosome inhibitors can be beneficial to restore Cav-1 expression in stromal tissues. There is an on-going clinical trial to test chloroquin therapy in DCIS patients (PINC, Preventing Invasive Breast Neoplasia with Chloroquine (Martinez-Outschoorn et al., 2010). Cav-1 is one of Src and Bcr-Abl substrates that can potentially be used for marker to predict therapeutic response of a substance targeting multitude kinases. Several studies have shown that elevated Cav-1 expression is marker for therapeutic response to desatinib (multikinase inhibitor) in breast, lung, liver and prostate cancer cells (Tryfonopoulos et al., 2011; Finn et al., 2013). Cav-1 expression has also been correlated with basal-type and triple negative breast cancer therefore Cav-1 expression can be used to select triple negative or basal-type breast cancer that might benefit from desatinib treatment. Thyrosine-14 phosphorylated caveolin leads to the increased sensitivity to paclitaxel through inhibition of Bcl-2. Some clinical trials have been conducted to evaluate increased sensitivity of paclitaxel through caveolin phosphorylation (ClinicalTrials NCT00046527, NCT00046514) (Shajahan et al., 2012).

Cavolin-1 plays an important role in the pathogenesis of breast cancer through multiple signaling pathways as summarized in Figure 1. Further studies are required to elucidate the detailed molecular networks and their potentials for diagnostic and prognostic markers as well as therapeutic response to chemotherapy and targeted therapy. Developing novel breast cancer therapy by exploiting caveolin-1 and caveolae is still challenging due to their roles in multiple signaling pathways and biological processes that presumably are cell- and tissue-specific. Therefore, systematic and comprehensive evaluation of Cav-1 expression and their molecular networks in different type breast cancers is required.

\section{Acknowledgements}

This study was supported by an extramural funding from Mochtar Riady Compresehtive Cancer Center (MRCCC) Hospital Siloam Semanggi Jakarta, Indonesia. The funding body did not have any role in the design and preparation of the manuscript.

\section{References}

Agelaki S, Spiliotaki M, Markomanolaki H, et al (2009). Caveolin-1 regulates EGFR signaling in $\mathrm{MCF}-7$ breast cancer cells and enhances gefitinib-induced tumor cell inhibition. Cancer Biol Ther, 8, 1470-7.

Bhowmick NA, Neilson EG, Moses HL (2004). Stromal fibroblasts in cancer initiation and progression. Nature, 432, 332-7.

Bonuccelli G, Whitaker-Menezes D, Castello-Cros R, et al 2010. The reverse Warburg effect: Glycolysis inhibitors prevent the tumor promoting effects of caveolin-1 deficient cancer associated fibroblasts. Cell Cycle, 9, 1960-71.

Bucci M, Gratton JP, Rudic RD, et al (2000). In vivo delivery of the caveolin-1 scaffolding domain inhibits nitric oxide synthesis and reduces inflammation. Nat Med, 6, 1362-7.

Burke P, Schooler K, Wiley HS (2001). Regulation of epidermal growth factor receptor signaling by endocytosis and intracellular trafficking. Mol Biol Cell, 12, 1897-1910. 
Sumadi Lukman Anwar et al

Cai C, Chen J (2004). Overexpression of caveolin-1 induces alteration of multidrug resistance in Hs578T breast adenocarcinoma cells. Int J Cancer, 111, 522-9.

Carroll JS, Meyer CA, Song J, et al (2006). Genome-wide analysis of estrogen receptor binding sites. Nat Genet, 38, 1289-97.

Castello-Cros R, Bonuccelli G, Molchansky A, et al (2011). Matrix remodeling stimulates stromal autophagy, "fueling" cancer cell mitochondrial metabolism and metastasis. Cell Cycle, 10, 2021-34.

Cirri P, Chiarugi P (2011). Cancer associated fibroblasts : the dark side of the coin. Am J Cancer Res, 1, 482-97.

Clemons M, Goss P (2001). Estrogen and the risk of breast cancer. NEJM, 344, 276-285.

Cohen AW, Razani B, Wang XB, et al (2003). Caveolin-1deficient mice show insulin resistance and defective insulin receptor protein expression in adipose tissue. Am J Physiol Cell Physiol, 285, C222-5.

Dong X, Mao S, Wen H (2013). Upregulation of proinflammatory genes in skin lesions may be the cause of keloid formation (Review). Biomed Rep, 1, 833-6.

El-Gendi SM, Mostafa MF, El-Gendi AM, et al (2012). Stromal caveolin-1 expression in breast carcinoma. Correlation with early tumor recurrence and clinical outcome. Pathol Oncol Res, 18, 459-69.

Elsheikh SE, Green AR, Rakha EA, et al (2008). Caveolin 1 and Caveolin 2 are associated with breast cancer basal-like and triple-negative immunophenotype. Br J Cancer, 99, 327-34.

Van den Eynden GG, Van Laere SJ, Van der Auwera I, et al (2006).Overexpression of caveolin-1 and -2 in cell lines and in human samples of inflammatory breast cancer. Breast Cancer Res Treat, 95, 219-28.

Fielding PE, Chau P, Liu D, Spencer TA, Fielding CJ, (2004). Mechanism of platelet-derived growth factor-dependent caveolin-1 phosphorylation: relationship to sterol binding and the role of serine-80. Biochemistry, 43, 2578-86.

Finn RS, Dering J, Ginther C (2007). Dasatinib, an orally active small molecule inhibitor of both the src and abl kinases, selectively inhibits growth of basal-type/"triple-negative" breast cancer cell lines growing in vitro. Breast Cancer Res Treat, 105, 319-26.

Finn RS, Aleshin A, Dering J, et al (2013). Molecular subtype and response to dasatinib, an Src/Abl small molecule kinase inhibitor, in hepatocellular carcinoma cell lines in vitro. Hepatology, 57, 1838-46.

Fiucci G, Ravid D, Reich R, Liscovitch M, et al (2002). Caveolin-1 inhibits anchorage-independent growth anoikis and invasiveness in MFC-7 human breast cancer cells. Oncogene, 21, 2365-2375.

Fujita Y, Maruyama S, Kogo H, Matsuo S, Fujimoto T (2004). Caveolin-1 in mesangial cells suppresses MAP kinase activation and cell proliferation induced by bFGF and PDGF. Kidney Int, 66, 1794-04.

Giusiano S, Cochet C, Filhol O, et al (2011). Protein kinase $\mathrm{CK} 2 \alpha$ subunit over-expression correlates with metastatic risk in breast carcinomas: Quantitative immunohistochemistry in tissue microarrays. Eur J Cancer, 47, 792-801.

Glait C, Ravid D, Lee SW, Liscovitch M, Werner H (2006). Caveolin-1 controls BRCA1 gene expression and cellular localization in human breast cancer cells. FEBS Lett, 580, 5268-74.

Goetz JG, Minguet S, Navarro-Lérida I, et al (2011). Biomechanical remodeling of the microenvironment by stromal caveolin-1 favors tumor invasion and metastasis. Cell, 146, 148-63.

Gupta R, Toufaily C, Annabi B (2014). Caveolin and cavin family members: dual roles in cancer. Biochimie, 107,
188-202.

Hayashi K, Matsuda S, Machida K, et al (2001). Invasion activating caveolin-1 mutation in human scirrhous breast cancers. Cancer Res, 61, 2361-64.

Hino M, Doihara H, Kobayashi K, Aoe M, Shimizu N (2003). Caveolin-1 as tumor suppressor gene in breast cancer. Surg Today, 33, 486-90.

Jezierska-Drutel A, Rosenzweig SA, Neumann CA, et al (2013). Role of oxidative stress and the microenvironment in breast cancer development and progression. Adv Cancer Res, 119, 107-25.

Joglekar M, Elbazanti WO, Weitzman MD, Lehma H, van Golen KL (2015). Caveolin-1 mediates inflamatory breast cancer cell invasion via the Akt1 pathway and RhoC GTPase. J Cell Biochem, 116, 923-33.

Kiss AL (2012). Caveolae and the regulation of endocytosis. Adv Exp Med Biol, 729, 14-28.

Koo JS, Park S, Kim SI, Lee S, Park BW (2011). The impact of caveolin protein expression in tumor stroma on prognosis of breast cancer. Tumour Biol, 32, 787-99.

Lajoie P, Nabi IR (2010). Lipid rafts, caveolae, and their endocytosis. Int Rev Cell Mol Biol, 282, 135-63.

Lavie Y, Fiucci G, Liscovitch M (2001). Upregulation of caveolin in multidrug resistant cancer cells: functional implications. Adv Drug Deliv Rev, 49, 317-23.

Lee H, Park DS, Razani B, et al (2002). Caveolin-1 mutations (P132L and null) and the pathogenesis of breast cancer: caveolin-1 (P132L) behaves in a dominant-negative manner and caveolin-1 (-/-) null mice show mammary epithelial cell hyperplasia. Am J Pathol, 161, 1357-69.

Lee SW, Reimer CL, Oh P, Campbell DB, Schnitzer JE, (1998). Tumor cell growth inhibition by caveolin re-expression in human breast cancer cells. Oncogene, 16, 1391-97.

Ma X, Liu L, Nie W, et al (2013). Prognostic role of caveolin in breast cancer: a meta-analysis. Breast, 22, 462-9.

Maldonado-Báez L, Williamson C, Donaldson JG (2013). Clathrin-independent endocytosis: A cargo-centric view. Exp Cell Res, 319, 2759-69.

Martinez-Outschoorn U, Sotgia F, Lisanti MP (2014). Tumor microenvironment and metabolic synergy in breast cancers: Critical importance of mitochondrial fuels and function. Semin Oncol, 41, 195-216.

Martinez-Outschoorn UE, Trimmer C, Lin Z, WhitakerMenezes D, et al (2010). Autophagy in cancer associated fibroblasts promotes tumor cell survival: Role of hypoxia, HIF1 induction and NFxB activation in the tumor stromal microenvironment. Cell Cycle, 9, 3515-33.

Martinez-Outschoorn UE, Whitaker-Menezes D, Lin Z, et al (2011). Cytokine production and inflammation drive autophagy in the tumor microenvironment: Role of stromal caveolin-1 as a key regulator. Cell Cycle, 10, 1784-93.

Martinez-Outschoorn UE, Whitaker-Menezes D, Lin Z, et al (2010). Tumor cells induce the cancer associated fibroblast phenotype via caveolin-1 degradation: Implications for breast cancer and DCIS therapy with autophagy inhibitors. Cell Cycle, 9, 2423-33.

Martinez-Outschoorn UE, Sotgia F, Lisanti MP, (2015). Caveolae and signalling in cancer. Nat Rev Cancer, 15, 225-37.

Mercier I, Camacho J, Titchen K, et al (2012). Caveolin-1 and accelerated host aging in the breast tumor microenvironment: Chemoprevention with rapamycin, an mTOR inhibitor and anti-aging drug. Am J Pathol, 181, 278-93.

Mercier I, Casimiro MC, Wang C, et al (2008). Human breast cancer-associated fibroblasts (CAFs) show caveolin-1 downregulation and RB tumor suppressor functional inactivation: Implications for the response to hormonal therapy. Cancer Biol Ther, 7, 1212-25. 
Mercier I, Lisanti M (2012). Caveolin-1 and breast cancer: a new clinical perspective. Adv Exp Med Biol, 729, 83-94.

Mercier I, Lisanti MP (2012). Caveolin-1 and breast cancer: a new clinical perspective. caveolins and caveolae: roles in signaling and disease mechanisms. Adv Exp Med Biol, 729, 83-94.

Mineo C, Gill GN, Anderson RGW, (1999). Regulated migration of epidermal growth factor receptor from caveolae. $J$ Biol Chem, 274, 30636-43.

Ørom UA, Lim MK, Savage JE, et al (2012). MicroRNA-203 regulates caveolin-1 in breast tissue during caloric restriction. Cell Cycle, 11, 1291-5.

Otranto M, Sarrazy V, Bonté F, et al (2012). The role of the myofibroblast in tumor stroma remodeling. Cell Adh Mig, 6, 203-19.

Park DS, Lee H, Frank PG, et al (2002). Caveolin-1-deficient mice show accelerated mammary gland development during pregnancy, premature lactation, and hyperactivation of the Jak-2/STAT5a signaling cascade. Mol Biol Cell, 13, 3416-30.

Park JH, Han HJ (2009). Caveolin-1 plays important role in EGFinduced migration and proliferation of mouse embryonic stem cells: involvement of PI3K/Akt and ERK. Am J Physiol Cell Physiol, 297, 935-44.

Park JH, Lee MY, Han HJ, et al (2009). A potential role for caveolin-1 in estradiol-17beta-induced proliferation of mouse embryonic stem cells: involvement of Src, PI3K/Akt, and MAPKs pathways. Int J Biochem Cell Biol, 41, 659-65.

Park SS, Kim JE, Kim YA, Kim YC, Kim SW (2005). Caveolin-1 is down-regulated and inversely correlated with HER2 and EGFR expression status in invasive ductal carcinoma of the breast. Histopathology, 47, 625-30.

Park WY, Park JS, Cho KA, et al (2000). Up-regulation of caveolin attenuates epidermal growth factor signaling in senescent cells. J Biol Chem, 275, 20847-52.

Patani N, Lambros MB, Natrajan R, et al (2012). Non-existence of caveolin-1 gene mutations in human breast cancer. Breast Cancer Res Treat, 131, 307-10.

Patani N, Martin LA, Reis-Filho JS, Dowsett M, (2012). The role of caveolin-1 in human breast cancer. Breast Cancer Res Treat, 131, 1-15.

Pavlides S, Tsirigos A, Migneco G, et al (2010). The autophagic tumor stroma model of cancer: Role of oxidative stress and ketone production in fueling tumor cell metabolism. Cell Cycle, 9, 3485-505.

White BP, Molloy MP, Zhao H, et al (2013). Extranuclear ERalpha is associated with regression of T47D PKCalphaoverexpressing, tamoxifen-resistant breast cancer. $\mathrm{Mol}$ Cancer, 12, 34.

Perrone G, Altomare V, Zagami M, et al (2009). Caveolin-1 expression in human breast lobular cancer progression. Mod Pathol, 22, 71-8.

Qian N, Ueno T, Kawaguchi-Sakita N, et al (2011). Prognostic significance of tumor/stromal caveolin-1 expression in breast cancer patients. Cancer Sci, 102, 1590-6.

Rao X, Evans J, Chae H, et al (2012). CpG island shore methylation regulates caveolin-1 expression in breast cancer. Oncogene, 32, 4519-28

Ren M, Liu F, Zhu Y, et al (2014). Absence of caveolin-1 expression in carcinoma associated fibroblast of invasive micropapollary carcinoma of the breast predicts poor patient outcome. Virchows Arch, 465, 291-8.

Pinilla SM, Honrado E, Hardisson D, Benítez J, Palacios J, (2006). Caveolin-1 expression is associated with a basal-like phenotype in sporadic and hereditary breast cancer. Breast Cancer Res Treat, 99, 85-90.

Sagara Y, Mimori K, Yoshinaga K, et al (2004). Clinical significance of Caveolin-1, Caveolin-2 and HER2/neu
mRNA expression in human breast cancer. Br J Cancer, 91, 959-65.

Savage K, Lambros MB, Robertson D, et al (2007). Caveolin 1 is overexpressed and amplified in a subset of basallike and metaplastic breast carcinomas: A morphologic, ultrastructural, immunohistochemical, and in situ hybridization analysis. Clin Cancer Res, 13, 90-101.

Schlegel A, Wang C, Katzenellenbogen BS, Pestell RG, Lisanti MP, (1999). Caveolin-1 potentiates estrogen receptor $\alpha$ $($ ER $\alpha)$ signaling. Caveolin-1 drives ligand-independent nuclear translocation and activation of ER $\alpha . J$ Biol Chem, 274, 33551-6.

Schlegel A, Wang C, Pestell RG, Lisanti MP, (2001). Ligandindependent activation of oestrogen receptor alpha by caveolin-1. Bioch J, 359, 203-10.

Sekhar SC, Kasai T, Satoh A, et al (2013). Identification of caveolin-1 as a potential causative factor in the generation of trastuzumab resistance in breast cancer cells. J Cancer, 4,391-401.

Senetta R, Stella G, Pozzi E, et al (2013). Caveolin-1 as a promoter of tumour spreading: When, how, where and why. J Cell Mol Med, 17, 325-36.

Shajahan AN, Dobbin ZC, Hickman FE, Dakshanamurthy S, Clarke R, (2012). Tyrosine-phosphorylated caveolin-1 (Tyr14) increases sensitivity to paclitaxel by inhibiting BCL2 and BCLxL proteins via c-Jun N-terminal Kinase (JNK). $J$ Biol Chem, 287, 17682-92.

Siegel R, Ma J, Zou Z, Jemal A, (2014). Cancer statistics, 2014. CA Cancer J Clin, 64, 9-29.

Simpkins SA, Hanby AM, Holliday DL, Speirs V, (2012). Clinical and functional significance of loss of caveolin-1 expression in breast cancer-associated fibroblasts. J Pathol, 227, 490-8.

Sloan EK, Ciocca DR, Pouliot N, et al (2009). Stromal cell expression of caveolin-1 predicts outcome in breast cancer. Am J Pathol, 174, 2035-43.

Sloan EK, Stanley KL, Anderson RL, (2004). Caveolin-1 inhibits breast cancer growth and metastasis. Oncogene, 23, 7893-7.

Sotgia F (2012). Caveolin-1 and Cancer Metabolism in the Tumor Microenvironment: Markers, Models, and Mechanisms. Annual Review of Pathology: Mechanisms of Disease, 7, 423-467.

Sotgia F, Martinez-Outschoorn UE, Howell A, et al (2006). Caveolin-1, mammary stem cells, and estrogen-dependent breast cancers. Cancer Res, 66, 10647-51.

Sotgia F, Martinez-Outschoorn UE, Pavlides S, et al (2011). Understanding the Warburg effect and the prognostic value of stromal caveolin-1 as a marker of a lethal tumor microenvironment. Br Cancer Res, 13, 213.

Syeed N, Husain SA, Abdullah S, et al (2010). Caveolin-1 promotes mammary tumorigenesis: Mutational profile of the Kashmiri population. Asian Pac J Cancer Prev, 11, 689-96.

Syeed N, Husain SA, Abdullah S, et al (2010). Mutational profile of the CAV-1 gene in breast cancer cases in the ethnic Kashmiri population. Asian Pac J Cancer Prev, 11, 1099-105.

Tagawa A, Mezzacasa A, Hayer A, et al (2005). Assembly and trafficking of caveolar domains in the cell: Caveolae as stable, cargo-triggered, vesicular transporters. J Cell Biol, 170, 769-79.

Thomas NB, Hutcheson IR, Campbell L, et al (2010). Growth of hormone-dependent MCF-7 breast cancer cells is promoted by constitutive caveolin-1 whose expression is lost in an EGF-R-mediated manner during development of tamoxifen resistance. Breast Cancer Res Treat, 119, 575-91.

Tian F, Wu H, Li Z, et al (2009). Activated PKCalpha/ERK1/2 signaling inhibits tamoxifen-induced apoptosis in C6 cells. 
Trimmer C, Sotgia F, Whitaker-Menezes D, et al (2011). Caveolin-1 and mitochondrial SOD2 (MnSOD) function as tumor suppressors in the stromal microenvironment: A new genetically tractable model for human cancer associated fibroblasts. Cancer Biol Ther, 11, 383-94.

Tryfonopoulos D, Walsh S, Collins DM, et al (2011). Src: a potential target for the treatment of triple-negative breast cancer. Ann Oncol, 22, 2234-40.

Wang XX, Wu Z, Huang HF, et al (2013). Caveolin-1, through its ability to negatively regulate TLR4, is a crucial determinant of MAPK activation in LPS-challenged mammary epithelial cells. Asian Pac J Cancer Prev, 14, 2295-9.

Wang Y, Yu J, Zhan Q (2008). BRCA1 regulates caveolin-1 expression and inhibits cell invasiveness. Biochem Biophys Res Commun, 370, 201-6.

Wang Z, Wang N, Li W, et al (2014). Caveolin-1 mediates chemoresistance in breast cancer stem cells via $\beta$-catenin/ ABCG2 signaling pathway. Carcinogenesis, 35, 2346-56.

Weigelt B, Geyer FC, Natrajan R, et al (2010). The molecular underpinning of lobular histological growth pattern: A genome-wide transcriptomic analysis of invasive lobular carcinomas and grade- and molecular subtype-matched invasive ductal carcinomas of no special type. J Pathol, 220, 45-57.

Williams TM, Medina F, Badano I, et al (2004). Caveolin-1 gene disruption promotes mammary tumorigenesis and dramatically enhances lung metastasis in vivo: Role of Cav-1 in cell invasiveness and matrix metalloproteinase (MMP-2/9) secretion. J Biol Chem, 279, 51630-46.

Williams TM, Lee H, Cheung MW, et al (2004). Combined loss of INK4a and caveolin-1 synergistically enhances cell proliferation and oncogene-induced tumorigenesis. Role of INK4a/CAV-1 in mammary epithelial cell hyperplasia. $J$ Bioll Chem, 279, 24745-56.

Williams TM, Lisanti MP, (2005). Caveolin-1 in oncogenic transformation, cancer, and metastasis. Am J Physiol Cell Physiol, 288, 494-506.

Williams TM, Lisanti MP, (2004). The caveolin proteins. Genome Biol, 5, 214.

Witkiewicz AK, Dasgupta A, Sotgia F, et al (2009). An absence of stromal caveolin-1 expression predicts early tumor recurrence and poor clinical outcome in human breast cancers. Am J Pathol, 174, 2023-34.

Witkiewicz AK, Kline J, Queenan M, et al (2011). Molecular profiling of a lethal tumor microenvironment, as defined by stromal caveolin-1 status in breast cancers. Cell Cycle, 10, 1794-1809.

Witkiewicz AK, Dasgupta A, Nguyen K, et al (2009). Stromal caveolin-1 levels predict early DCIS progression to invasive breast cancer. Cancer Biol Ther, 8, 1071-9.

Wu P, Qi B, Zhu H, et al (2007). Suppression of staurosporinemediated apoptosis in Hs578T breast cells through inhibition of neutral-sphingomyelinase by caveolin-1. Cancer Lett, 256, 64-72.

Zagouri F, Sergentanis TN, Chrysikos D, Filipits M, Bartsch $\mathrm{R}$, (2012). mTOR inhibitors in breast cancer: A systematic review. Gynecol Oncol, 127, 662-72.

Zhang EY, Cristofanilli M, Robertson F, et al (2013). Genome wide proteomics of ERBB2 and EGFR and other oncogenic pathways in inflammatory breast cancer. J Proteome Res, 12, 2805-17.

Zou W, McDaneld L, Smith LM, (2003). Caveolin-1 haploinsufficiency leads to partial transformation of human breast epithelial cells. AntiCancer Res, 23, 4581-6. 